\title{
A Diabetes-susceptible HLA Haplotype Is Best Defined by a Combination of HLA-DR and -DQ Alleles
}

\author{
Michael J. Sheehy, ${ }^{\star}$ Stephen J. Scharf, ${ }^{\star}$ James R. Rowe," Mabel H. Neme de Gimenez, Louise M. Meske," \\ Henry A. Erlich, ${ }^{\ddagger}$ and Barbara S. Nepom \\ * Research Department, American Red Cross Blood Services, and the Department of Medicine, University of Wisconsin, Madison, \\ Wisconsin 53705; ${ }^{\ddagger}$ Department of Genetics, Cetus Corporation, Emeryville, California 94608; and ${ }^{\S}$ Genetic Systems, Inc. \\ and the Virginia Mason Research Center, Seattle, Washington 98101
}

\begin{abstract}
HLA-DR4 is associated with insulin-dependent diabetes mellitus (IDDM) in many populations. Many recent studies suggest that the DR4 effect is really due to DQ3.2, an allele of the nearby DQB1 locus. We used T cell clones, MAb, and allelespecific oligonucleotides to test IDDM and control subjects for DR4 subtypes (Dw4, Dw10, Dw13, and Dw14) and for DR4associated DQB1 alleles (DQ3.1 and DQ3.2). We find that (a) IDDM is approximately equally associated with alleles of the DRB1 locus (Dw4 and Dw10, combined relative risk, $R R$ = 6.4) and the DQB1 locus (DQ3.2, RR = 5.9); and (b) there is significant interaction, in a statistical sense, between these $D R$ and DQ alleles in IDDM. The only IDDM-associated DR4 haplotypes were those carrying the IDDM-associated alleles at both loci $(R R=12.1)$; haplotypes with Dw4 or 10 but not DQ3.2, or vice versa, had a $R \boldsymbol{R}<1$. Alternative explanations include: $(a)$ that susceptibility requires specific allelic products of both DR and DQ loci; (b) that the combination of certain DR and DQ alleles marks haplotypes with the true susceptibility allele at a third locus; or (c) that Dw4 and 10 mark haplotypes with an allele at another locus that interacts with DQ3.2. As discussed, this third locus is unlikely to be DQA1 (DQ $\alpha)$. The data thus are not easily reconciled with an exclusive effect of HLA-DQ. This information increases our ability to predict IDDM by genetic typing: in the population studied, heterozygotes DR3/[DQ3.2, Dw4] or DR3/[DQ3.2, Dw10] had a relative risk of 38.0 and an absolute risk of 1 in 15.
\end{abstract}

\section{Introduction}

Like many autoimmune diseases, insulin-dependent diabetes mellitus (IDDM) ${ }^{1}$ is associated with particular HLA alleles. The most consistent associations have been reported with HLA-DR3 and DR4, and especially with the heterozygous genotype DR3/4 $(1,2)$.

Recent papers from our laboratories (3-5) and others (6-15) show that some DR4 haplotypes are IDDM associated,

Address reprint requests to Dr. Michael J. Sheehy, American Red Cross Blood Services, 4860 Shegoygan Ave., Madison, WI 53705. 1988.

Received for publication 21 July 1988 and in revised form 7 October

1. Abbreviations used in this paper: IDDM, insulin-dependent diabetes mellitus; NOD, nonobese diabetic; $R R$, relative risk.

J. Clin. Invest.

(c) The American Society for Clinical Investigation, Inc.

0021-9738/89/03/0830/06 \$2.00

Volume 83, March 1989, 830-835 whereas others are not. DR4 thus may be merely a marker for the true diabetes gene(s). The goal of this study was to better define the IDDM-associated DR4 haplotype(s), so as to better identify the individuals at risk for IDDM.

DR4, a serologically defined allele of the DRB1 locus (16), has five defined subtypes (Dw4, Dw10, and Dw13-15) and undefined subtype(s) (Dw-blank), all distinguishable by reactions of $T$ lymphocytes in mixed leukocyte culture (17). Several studies have shown the Dw4 subtype to be IDDM associated $(11,18,19)$, whereas Suciu-Foca et al. (6) found a significant increase in Dw10 but not Dw4. In a carefully controlled study of 106 IDDM patients and 107 controls from southern Wisconsin and 76 patients and 49 controls from the Boston area (20), we found that both Dw4 and Dw10 were significantly IDDM associated, with relative risks $(R R)$ of 6.2 and 7.0 respectively, whereas the remaining DR4 subtypes were not IDDM associated $(R R$ for Dw14 $=1.1$; and $R R$ for Dw13, Dw15 and Dw-blank, combined $=0.4$ ). Therefore, in this study, we focused on Dw4 and Dw10.

"DQ3.2," an allele of the DQB1 locus (initially identified by leukocyte testing with monoclonal or polyclonal antibodies and by DNA testing with Southern blots), also identifies a diabetes-associated subset of DR4-bearing haplotypes. We and others $(4,5,7,9,12-15)$ find that $\sim 95 \%$ of DR4-positive IDDM patients, but only $\sim 65-70 \%$ of DR4-positive control subjects, have the DQ3.2 allele.

Alleles at either the DRB1 locus (Dw4 and Dw10) or the DQB1 locus (DQ3.2) thus could account for the DR4 association with IDDM. More studies have demonstrated the DQ3.2 association with IDDM, but this could be due to the greater ease of detecting DQ3.2 by the methods commonly used (see Discussion).

To determine which locus is more likely to play a causal role in IDDM, we tested DR4-positive IDDM and control subjects with human $T$ cell clones (to identify DR4 subtypes), with MAb (to identify DQw3 subtypes) and with allele-specific oligonucleotide probes (for both DR4 and DQw3 subtypes). Among other analyses, we used the type of analysis used by Platz et al. (19) to show that HLA-B/IDDM associations are due to linkage disequilibrium between HLA-B and DR. Studying 69 DR4-positive subjects (40 patients and 29 controls), we controlled for DRB1 alleles and tested whether there remains an IDDM association with DQ3.2. Likewise we controlled for DQB1 alleles and tested whether there remains an IDDM association with Dw4 and Dw10.

For all statistical analyses in this study, Dw4 and Dw10 data were pooled, since our intent was to determine which locus (DRB1 or DQB1) has the primary influence in IDDM, not to distinguish the effects of the two IDDM-associated alleles at the DRB1 locus. Similar results (not shown) were obtained when Dw4 data were analyzed alone, although the cor- 
responding $P$ values were slightly higher. Dw10, a DR4 subtype largely limited to Ashkenazi Jews, was infrequent in the southern Wisconsin population studied here ( 2 of 40 DR4positive IDDM patients and 0 of 29 DR4-positive control subjects). The Dw10 data, if analyzed in isolation from Dw4, thus would not be statistically significant.

\section{Methods}

Subjects. Patients $(n=195)$ and controls $(n=114)$ were carefully matched for geographic origin. All patients had classical ketosis-prone insulin-dependent diabetes. 54 patients we re from the University of Wisconsin Pediatric Diabetes Clinic (Dr. Michael MacDonald, Department of Pediatrics, University of Wisconsin, Madison) and 141 were from the Wisconsin Diabetes in Youth Study (Dr. Donn D'Alessio, Department of Preventive Medicine, University of Wisconsin, Madison). Controls were intended to represent the population, and were not selected to either include or exclude IDDM patients or their relatives. 55 controls were Wisconsin-born adults unselected with regard to age, sex, or family history of diabetes. Individuals born outside Wisconsin were specifically excluded. Each of the remaining 59 controls was an age strata- and sex-matched friend control for one of the patients from the Wisconsin Diabetes in Youth Study. To prevent attrition or depletion of cell samples, we made immortal cell lines from all subjects (see "Cells used"). Of these 309 subjects, 69 DR4-positive subjects (40 patients and 29 controls) were randomly selected and studied more intensively for DR4-related HLA alleles.

Cells used. Cells studied were 309 HLA-typed lymphoblastoid cell lines produced in M. J. Sheehy's laboratory. Cell lines were HLA-DR typed with antiserum trays from One Lambda Co. (Los Angeles, CA), the complement being diluted $1: 2$ to $1: 3$ with heat-inactivated complement.

Typing for Dw4 and Dw10 with T cell clones. Dw4 and Dw10, two of five defined subtypes of DR4, were determined by proliferation of human $T$ cell clones, Dw4 by clone M1-72 $(3,20)$ and Dw 10 by clone B34-85 (21). This testing is described in detail elsewhere (20). Results were classified as positive, negative, or ambiguous by cluster analysis as described by Shaw et al. (22). These two clones gave no ambiguous results in the experiments reported here.

Typing for subtypes of $D Q w 3$ with $M A b$. DQ3.2, a subtype of HLA-DQw3, was defined by indirect immunofluorescence using a FACS IV cell sorter. Each antibody was used as ascites fluid diluted $1: 160$, and the second antibody was affinity-purified fluorescein isothiocyanate-conjugated rabbit anti-mouse IgG. MAb used were: 17.15 (23), which binds to DQw3; A-10-83 (24), which binds to DQ3.1; and IIB3 (25), which binds to DQw1 and DQ3.2 (26). On cells heterozygous for DQw3 (either DQw1/w3 or DQw2/w3), the first two antibodies were used to identify DQ3.2 cells (positive for DQw3 but negative for DQ3.1). On cells that were homozygous DQw3/w3, MAb IIB3 was used to identify the DQ3.2-positive cells.

Oligonucleotide typing. From 1- $\mu \mathrm{g}$ DNA samples, the second exons encoding the polymorphic outer domains of the HLA-DRB1 and HLA-DQB1 chains were amplified by polymerase chain reaction, using specific DNA flanking primers as described $(27,28)$. Amplified DNA samples were dot blotted onto nylon membranes, and alleles were identified by autoradiography after hybridization with ${ }^{32} \mathrm{P}$-endlabeled oligonucleotide probes. Oligonucleotide sequences for the DQ3.1, DQ3.2, Dw4, Dw10, and Dw14 probes were described (27). The Dw13 probe sequence is CAGAGGCGGGCCGAGGT. The Dw10 probe cross-hybridizes to the DRB1 alleles of some DRw6 haplotypes; probe reactivity can be assigned to either the DR4 or DRw6 haplotype by restriction enzyme digestion of the amplified DNA (27). Similarly, the Dw14 probe cross-hybridizes to the DRB1 allele of the DR1 haplotype.

Blind testing. Immunological tests for Dw and $\mathrm{DQ}$ antigens were done in separate laboratories (M. J. Sheehy and B. S. Nepom, respectively), each without knowledge of the other's results. Confirmatory dot blot studies were done on randomized, blind coded samples in a third laboratory (H. A. Erlich).

Data analysis. Relative risk $(R R)$ is an odds ratio: (no. patients with antigen/no. patients without antigen)/(no. controls with antigen/no. controls without antigen) (29). Etiologic fraction is calculated as (frequency of antigen in patients - frequency of antigen in controls)/(1 - frequency of antigen in controls) (30). $P$ values were calculated by the Fisher Exact Test (31) for relationships of HLA alleles to IDDM and by log-linear analysis (32) for interaction between DR (Dw) and DQ alleles in IDDM.

\section{Results}

To determine if the association of HLA-Dw4 and 10 with IDDM is due to linkage disequilibrium with DQ3.2, or viceversa, we tested a single set of subjects for both DR4 subtypes and DQw3 subtypes. These markers were identified in all subjects at both the protein level in immunological assays (using MAb for DQw3 subtypes and T cell clones for DR4 subtypes) and the DNA level (by oligonucleotide probe analysis of specifically amplified DNA). All testing was done in blind fashion as described in Methods.

We previously reported (20) that only two of the DR4 subtypes, Dw4 and Dw10, are IDDM associated ( $R R$ of 6.2 and 7.0) in the population studied here. The $R R$ for Dw 14 was only 1.1 , and the $R R$ for Dw13, Dw15, and Dw blank, combined, was $<1$. We thus determined before starting this study that Dw4 and Dw10 data would be pooled (except for Table I, which summarizes the test results) and analyzed separately from the remaining DR4 subtypes.

Table I summarizes allelic assignments for the 69 selected DR4-positive cell lines. As described in Methods, both immunological tests and DNA dot blots were done for the alleles of primary interest (Dw4, Dw10, DQ3.1, and DQ3.2), whereas testing for Dw 13 and Dw14 was done by DNA dot blots alone.

Log-linear analysis of Dw $\times \mathrm{DQ} \times$ IDDM shows that $\mathrm{Dw}$ and DQ in combination play a role in IDDM susceptibility $(P$ $<0.05)$. In particular, the alleles DQ3.2 and Dw $(4+10)$ are preferentially associated in IDDM patients compared with controls.

The association of Dw4 and 10 with IDDM, shown here and described previously, cannot be due to linkage disequilibrium with DQ3.2 in the population. Dw4 and 10 were negatively correlated with DQ3.2 in the control group $(r=-0.44$ $\left.\left[\chi^{2}=3.86, P=0.045\right]\right)$. Only in the patient group was there a slight but nonsignificant positive correlation between the alleles at these two loci $\left(r=+0.20\left[\chi^{2}=0.08, P=0.64\right]\right)$.

The relationships of various DR4 haplotypes are summarized in Fig. 1. The IDDM-associated DR4 haplotype thus is best defined by a combination of Dw and DQ markers (Fig. 1 and Table II). Individuals that were either Dw4, DQ3.2 or Dw10, DQ3.2 made up 80\% of DR4-positive patients but only $28 \%$ of DR4-positive controls $\left(P=1.5 \times 10^{-5}\right.$, top section of Table II). Among all subjects (patients and controls with or without DR4, bottom section of Table II), the $R R$ for DR4 was 4.4. The DR4-associated DRB1 and DQB1 alleles had $R R \mathrm{~s}$ of 6.4 and 5.9, respectively. However, the risk associated with each locus is entirely attributable to haplotypes having IDDM-associated alleles at both loci $(R R=12.1)$. Haplotypes with the IDDM-associated allele at one locus but not the other had $R R<1$.

Together, the heterozygous phenotypes DR3/Dw4, DQ3.2 and DR3/Dw10, DQ3.2 (last line of Table II) had a $R R$ of 38 . 
Table I. Distribution of DR4-Positive Patients and Controls among Subtypes of DR4 and DQw3

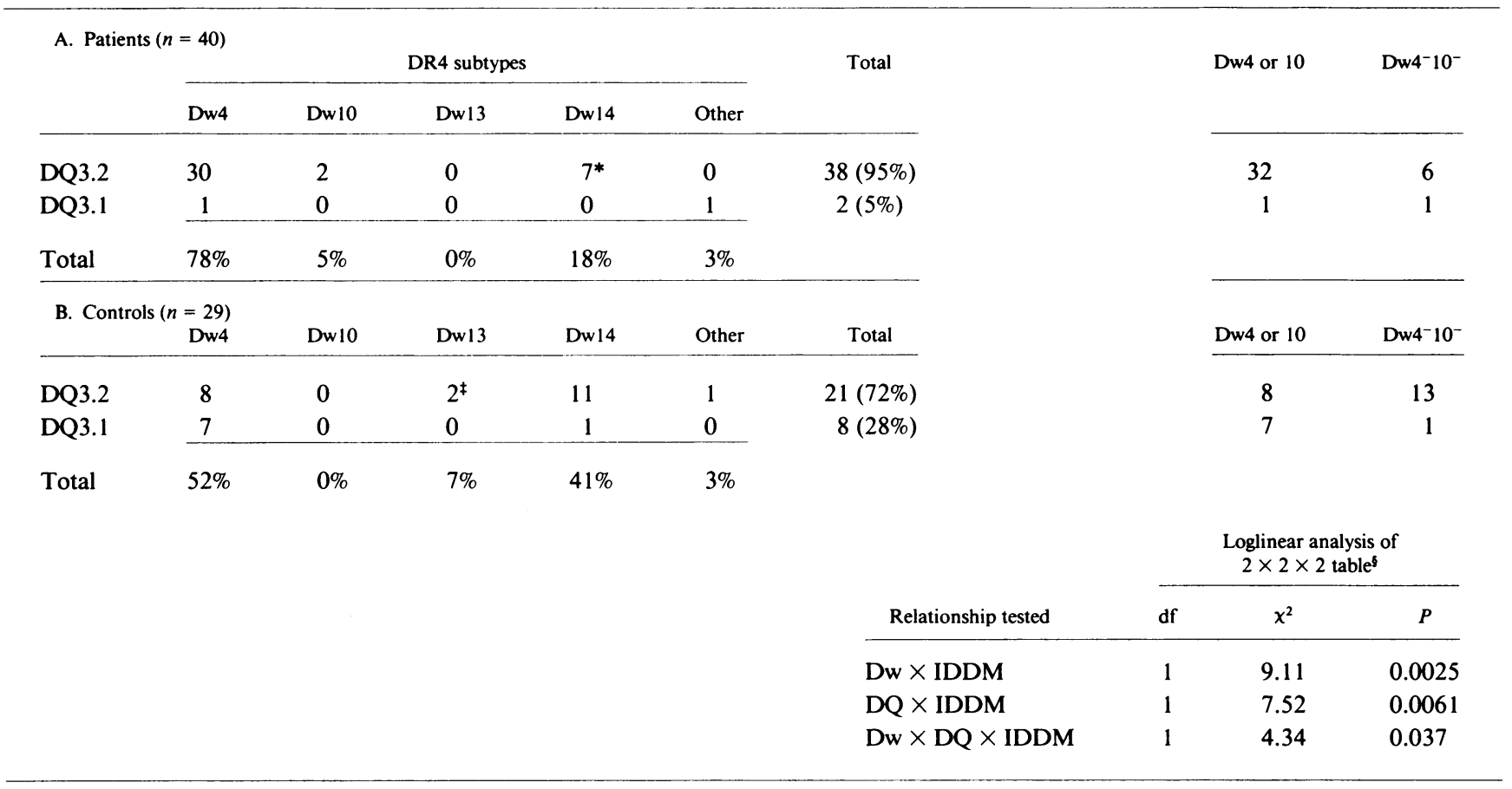

Typing for Dw4, Dw10, DQ3.1, and DQ3.2 was done both by immunological methods (T cell clone proliferation for Dw4 and 10 and MAb binding for DQ3.1 and 3.2) and by dot blots of specifically amplified DNA (all four of these alleles). Typing for Dw13 and Dw14, which were not explicitly included in our data analysis (Tables I and II), was done by DNA dot blots only. For Dw4, 67 of 69 results were concordant by immunological and DNA testing. One discrepant result was $+/-$ and one was $-/+$ (immunological/DNA results). Results for Dw10 were $100 \%$ concordant by the two methods. DQ3.2 results were exactly like those for Dw4, 67/69 concordant with one discrepancy in each direction. Discrepancies were resolved as follows: Dw4: discrepancies were resolved in favor of the $\mathrm{T}$ cell clone, because $\mathrm{T}$ cell proliferation is more likely than DNA blotting to be functionally relevant. DQ3.2: discrepancies were resolved in favor of the positive result. The $-/+$ sample was DQw3 positive with both polyclonal and MAbs, and reacted with the DQ3.2 DNA probe, but was nonreactive with both DQ3.1 and DQ3.2 antibodies. Similarly, the $+1-$ sample was DQw3 positive, reacted with the DQ3.2 antibody, but was nonreactive with both DQ3.1 and DQ3.2 DNA probes. The DQ3.2 DNA probe used corresponds to a part of the DQ molecule predicted to lie inside the antigen-binding pocket, and thus expected to be relevant in antigen presentation, whereas the antibody probably binds to a portion outside the antigen-binding pocket (Ucla, C., J. Gorski, B. Mach, and M. J. Sheehy, manuscript submitted for publication). A negative result with the DNA probe is probably less significant than a positive result, however, as the lack of binding could result from a silent mutation leaving the amino acid sequence unaltered. ${ }^{*}$ One Dw14-positive patient was also Dw4-positive (DR4 homozygous). ${ }^{\ddagger}$ One Dw13-positive control was also Dw4-positive (DR4 homozygous). $\S 2 \times 2 \times 2$ table consists of the eight numbers (two sets of four) set out to the right, in the top portion of Table I, with subjects divided into $($ IDDM [+ or -$]) \times($ Dw4 or $10[+$ or -$]) \times($ DQ3.2 [+ or -$])$.

Only $22 \%$ of the patients had these phenotypes, however, emphasizing the diversity of HLA types that can occur in IDDM patients despite the existence of certain highly susceptible types.

\section{Discussion}

Many studies, cited earlier, show that a DQB1 allele, DQ3.2, identifies an IDDM-associated subset of DR4 haplotypes. It

All DR4 Haplotypes

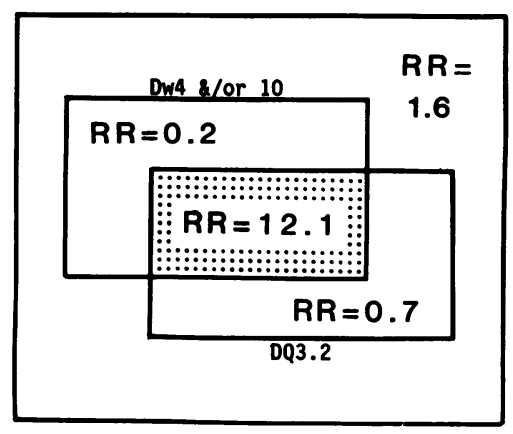

appeared, therefore, that DQ3.2 might be solely responsible for the DR4 association with IDDM. This clearly is only part of the story, as shown here by examining the relationships of DR4 subtypes (Dw4, Dw10, Dw13, Dw14, and Dw-undefined) to DQ3.2 among IDDM patients and controls. Table I shows that DQ3.2 haplotypes with Dw4 or Dw10 are increased in frequency in the DR4-positive patients compared with DR4-positive controls (75 vs. $28 \%$ for Dw4-DQ3.2 and 5 vs. 0\% for Dw10-DQ3.2), whereas DQ3.2 haplotypes with other DR4 subtypes were decreased in frequency in the DR4-positive patients compared with DR4-positive controls (0 vs. $7 \%$ for Dw13-DQ3.2, 18 vs. $38 \%$ for Dw14-DQ3.2, and 0 vs. $3 \%$ for Dw-undefined-DQ3.2).

We reiterate that the pooling of Dw4 and Dw10 data, and likewise the pooling of data for the remaining DR4 subtypes, were planned even before we began this study, because both Dw4 and Dw10 (but not other DR4 subtypes) were IDDM-associated in our Wisconsin and Boston studies (20). Similarly, in DNA sequence studies in the Berkeley, CA area (Scharf, S. J., and H. A. Erlich, unpublished observations), of seven IDDM patients typed as DR4, four were Dw 10 and three were Dw4. Although the discussion below refers to Dw4-DQ3.2 and 


\begin{tabular}{|c|c|c|c|c|c|}
\hline \multicolumn{6}{|c|}{ A. Frequencies among DR4-positive subjects } \\
\hline \multicolumn{2}{|c|}{ Dw4 or Dw10 } & \multicolumn{2}{|c|}{$83 \%$} & $52 \%$ & 0.007 \\
\hline \multicolumn{2}{|c|}{ DQ3.2 } & 95 & \multicolumn{2}{|c|}{$72 \%$} & 0.011 \\
\hline \multicolumn{2}{|c|}{ Dw4/DQ3.2 or Dw10/DQ3.2 } & \multicolumn{2}{|c|}{$32 / 40=80 \%$} & $8 / 29=28 \%$ & 0.00002 \\
\hline \multicolumn{6}{|c|}{ B. Frequencies among all subjects } \\
\hline & & Patients & Controls & Relative risk & Etiologic fraction \\
\hline DR3 & & $98 / 195=50 \%$ & $24 / 114=21 \%$ & 3.8 & 0.37 \\
\hline DR4 & & $127 / 195=65 \%$ & $34 / 114=30 \%$ & 4.4 & 0.50 \\
\hline \multicolumn{6}{|c|}{ Subsets of DR4 haplotypes ${ }^{\ddagger}$} \\
\hline Dw4 or Dw10 & & $54 \%$ & $15 \%$ & 6.4 & 0.45 \\
\hline DQ3.2 & & $62 \%$ & $22 \%$ & 5.9 & 0.51 \\
\hline Dw4 or Dw10 & DQ3.2 & & & & \\
\hline+ & + & $52 \%$ & $8 \%$ & 12.1 & 0.48 \\
\hline+ & - & $1.6 \%$ & $7.2 \%$ & 0.2 & $<0$ \\
\hline- & + & $9.8 \%$ & $13 \%$ & 0.7 & $<0$ \\
\hline- & - & $1.6 \%$ & $1.0 \%$ & 1.6 & 0.006 \\
\hline DR3/[Dw4 or 10 & & $22 \%$ & $0.7 \%$ & 38.0 & 0.21 \\
\hline
\end{tabular}

* Fisher exact test. $\quad{ }^{\ddagger}$ Percentages for subsets of DR4 haplotypes in part B were calculated from the frequencies of DR4 among all subjects (127 of 195 patients and 34 of 114 controls) and the frequencies of the indicated subsets among the 69 tested DR4-positive subjects (from Tables I and II). ${ }^{\S}$ Heterozygotes with one DR3 haplotype, and a DR4 haplotype bearing either Dw4 + DQ3.2 or Dw10 + DQ3.2.

Dw10-DQ3.2 as IDDM-associated HLA haplotypes, bear in mind that Dw10 was very infrequent in the population studied, even in the patient group. Any observations regarding Dw 10, if analyzed in isolation from Dw4, thus would not have been statistically significant.

HLA typing has been much less useful for predicting IDDM in the population than in affected families. Maclaren et al. (33) reported that the absolute risk of IDDM for DR3/4 heterozygotes in the population is $\sim 1$ in 40 , significantly less than the risk for HLA-identical siblings of IDDM patients (one in seven overall and one in four when the shared DR type is DR3/4). Similar sibling figures were obtained by Cavender et al. (34). Thus DR typing is 6-10 times less powerful as a predictor of IDDM in the population than among patients' siblings. The detection of a highly diabetogenic subset of DR4 haplotypes (Dw4-DQ3.2 and possibly Dw10-DQ3.2), described here, permits much greater predictive power as illustrated below.

In the same southern Wisconsin counties studied here, Klein et al. (35) found diabetes mellitus (types 1 and 2 not differentiated) in 2.3 per 1,000 or 1 in 435 persons age $20-29$, a figure close to the 1 in 400 reported by Maclaren et al. (33) for IDDM. The prevalence figure from Klein et al. is probably a slight underestimate of the risk for IDDM in the population tested, but will be used here for lack of specific prevalence data for IDDM. To the degree that this figure is too low, the calculations below will be conservative.

Using Bayes' theorem, the absolute risk of IDDM for any given HLA type is: $P($ IDDM $\mid$ HLA type $)=P($ IDDM $)$ $\times P($ HLA type $\mid$ IDDM $) / P($ HLA type). $P($ IDDM $) \geq 0.0023$ from Klein et al. (35). $P$ (HLA type/IDDM) is estimated by HLA frequencies in our patient group. Because our control group was selected to represent the population (controls selected without regard to IDDM in themselves or their rela- tives), $P$ (HLA type) is estimated by HLA frequencies in our control group.

The frequencies of DR3/4 heterozygosity in our patient and control groups were 0.27 and 0.026 , respectively (not shown) so, in the population studied, $P(I D D M \mid D R 3 / 4)$ is estimated as $0.0023 \times 0.27 / 0.026=0.024$, or 1 in 42 . This is very close to the 1 of 40 figure of Maclaren et al. (33). Because the DR4 haplotypes Dw4-DQ3.2 and Dw10-DQ3.2 made up $80 \%$ of DR 4 haplotypes in patients and $28 \%$ in controls (Table I), the absolute risk for persons heterozygous DR3/Dw4DQ3.2 or DR3/Dw10-DQ3.2 is estimated as $0.0023 \times 0.80$ $(0.27) / 0.28(0.026)=0.067$, or 1 in 15 .

The ability to identify persons with a genetic risk of 1 in 15 for IDDM makes it worthwhile to consider population screening for IDDM susceptibility. Persons identified as genetically susceptible could then be screened for immunological markers (antibodies to insulin and/or islet cells) that precede overt IDDM. These genetic and immunological tests will probably be important components of the effort to prevent IDDM. Possible preventive treatments are under investigation elsewhere.

There are several possible reasons why the absolute risk (1 in 15) for the HLA phenotypes DR3/Dw4-DQ3.2 and DR3/ Dw10-DQ3.2 in the population is less than that for patients' HLA-identical siblings (one in seven on average, one in four when both siblings are DR3/4): (a) we still may not have adequately defined the susceptible DR4 haplotypes; (b) DR3 haplotypes may be heterogeneous with regard to IDDM susceptibility; (c) the HLA-identical siblings share, on the average, half of their non-HLA genes as well, and some of these may influence IDDM susceptibility; and $(d)$ the siblings share a similar environment.

Among published studies attempting to subdivide DR4 haplotypes into those that do or do not confer IDDM susceptibility, many more have shown an association with DQB1 than 
with Dw alleles. IDDM also seems to correlate with certain DQB1 alleles on non-DR4 haplotypes $(13,36,37)$. Particularly striking is the negative correlation of IDDM with aspartate at amino acid position 57 of the DQB1 chain $(36,37)$. Thus, when we performed this study, we expected to find that the DRB1 associations (Dw4 and Dw10) with IDDM are due to linkage disequilibrium with DQ3.2. Instead, we found a highly significant association of Dw4 and 10 with IDDM, even among patients and controls that are DQ3.2-positive (32 of 38 $=84 \%$ of DQ3.2 $2^{+}$patients and 8 of $21=38 \%$ of DQ3.2 $2^{+}$ controls; $P=0.0005$ ). The IDDM association of Dw4 and 10 was slightly stronger than that for DQ3.2, whereas the etiologic fraction was slightly higher for DQ3.2.

If DRB1 alleles are as important as DQB1 alleles in explaining the DR4 effect in IDDM, it is curious that there are many more reports of IDDM association with DQB1 alleles. In our opinion, this is most likely attributable to the means to identify alleles of the two loci. Most of the recent studies of HLA and IDDM have emphasized leukocyte serology or DNA restriction fragment (Southern blot) analysis. Because no antibodies or restriction fragments have been found that distinguish the various DR4 subtypes, such studies would not detect any association that might exist between IDDM and the DR4 subtypes. In contrast, many restriction fragment length polymorphism (reviewed by Nepom et al. [38]) and several monoclonal and polyclonal antibodies, including those used here, distinguish DQ3.1 and 3.2. Until very recently, the only way to distinguish the various DR4 subtypes was mixed leukocyte culture testing with homozygous typing cells. This procedure is highly labor intensive, and the cellular reagents used are necessarily highly polyspecific.

At face value, the results presented here suggest that certain alleles at both DRB1 and DQB1 loci are needed for maximum susceptibility to IDDM. Alternatively, the combination of Dw4 or Dw10 with DQ3.2 may mark haplotypes with a diabetes susceptibility allele at a third locus nearby, or Dw4 and 10 may mark haplotypes with an allele at another locus that interacts with DQ3.2. The DQA1 (formerly DQ $\alpha$ ) locus, situated between DRB1 and DQB 1 on chromosome 6 , would be a reasonable candidate for this third locus, except that the deduced first-domain amino acid sequences did not vary among DQA1 alleles of four cell lines carrying Dw4, Dw10, Dw14, and Dw15 (39). Other explanations are also possible, but seem even less consistent with the available data.

Data from the nonobese diabetic (NOD) mouse are consistent with the first hypothesis. In the NOD strain, two genes on the same $\mathrm{H}-2$ haplotype (murine equivalent of HLA) seem to be required for IDDM susceptibility: a null allele at the IE-beta locus (HLA-DRB equivalent) and an allele at another locus, probably in the IA subregion (DQ equivalent) (40). Other alleles at both loci were strongly protective.

A similar mechanism may be operant in the human DR4 haplotype: i.e., Dw4 and possibly Dw10 at the DRB1 locus, and DQ3.2 at the DQB1 locus, may be among the least protective alleles for IDDM, at their respective loci. If this were true, maximum susceptibility would require non-protective alleles at both loci. We further postulate that the contribution of DR3, in DR3/4 heterozygotes, is due not only to a nonprotective DQB1 allele $(36,37)$ but also to a nonspecific enhancement of the immune response (41) whose mechanism is still unknown.
As stated above, this is only one of several possible models to explain our data, although it seems to us the simplest model compatible with all the available data. As discussed before (20), however, Dw4 and Dw10 are relatively dissimilar among the five DR4 subtypes that have been sequenced (Dw4, Dw10, Dw13, Dw14, and Dw15), whereas Dw4 and Dw14 are very similar. We thus lack a simple structural basis to explain why Dw4 and 10 should be uniquely nonprotective for IDDM.

The results reported here may account for the observation of MacDonald et al. (42) that parent-child pairs with IDDM share DR4 much more often than DR3. DR3 has a relative risk of 3.8 in the population tested here (Table II), whereas the diabetes-associated subset of DR4 haplotypes (the subset likely to be shared by affected parent-child pairs) has a $R R$ of 12.1 .

\section{Acknowledgments}

We thank Dr. Frits Koning for MAb IIB3, Dr. Michael MacDonald and Dr. Donn D'Alessio for patient and control blood samples, Ms. Eva Kubinski and Mr. Matthew Hanson for assistance with HLA-DR typing and cell culture, respectively, and Corey Levinson, Dragan Spasic, and Laurie Goda for synthesis of oligonucleotides used for oligonucleotide DNA typing.

This work was supported in part by National Institutes of Health grants DK-38209 and DK-33232, March of Dimes grant 5-538, Juvenile Diabetes Foundation grant 187699 , and a matching grant from the American Red Cross. M. J. Sheehy is an Established Investigator of the American Red Cross.

\section{References}

1. Svejgaard, A., P. Platz, and L. P. Ryder. 1983. HLA and disease 1982: a survey. Immunol. Rev. 70:193-218.

2. Rotter, J. I., C. Anderson, R. Rubin, J. E. Congleton, P. I. Terasaki, and D. C. Rimoin. 1983. HLA genotypic study of insulindependent diabetes: the excess of DR3/DR4 heterozygotes allows rejection of the recessive hypothesis. Diabetes. 32:169-174.

3. Sheehy, M. J., J. R. Rowe, and M. J. MacDonald. 1985. A particular subset of HLA-DR4 accounts for all or most of the DR4 associations in insulin-dependent diabetes. Diabetes. 34:942-944.

4. Nepom, B. S., J. Palmer, S. J. Kim, J. A. Hansen, S. L. Holbeck, and G. T. Nepom. 1986. Specific genomic markers for the HLA-DQ subregion discriminate between $\mathrm{DR}^{+}$insulin-dependent diabetes mellitus and DR4 ${ }^{+}$seropositive juvenile rheumatoid arthritis. J. Exp. Med. 164:345-350.

5. Arnheim, N., C. Strange, and H. Erlich. 1985. Use of pooled DNA samples to detect linkage disequilibrium of polymorphic restriction fragments and human disease: studies of the HLA class II loci. Proc. Natl. Acad. Sci. USA. 82:6970-6974.

6. Suciu-Foca, N., P. Rubinstein, J. Nicholson, E. Susinno, J. Weiner, M. Godfrey, M. Hardy, E. Rayfield, and K. Reemtsma. 1979. Juvenile diabetes mellitus and the HLA system. Transplant. Proc. 11:1309-1313.

7. Owerbach, D., Å. Lernmark, P. Platz, L. P. Ryder, L. Rask, P. Peterson, and J. Ludvigsson. 1983. HLA-D region $\beta$-chain DNA endonuclease fragments differ between HLA-DR identical healthy and insulin-dependent diabetic individuals. Nature (Lond.) 303:815-817.

8. Cudworth, A. G., and E. Wolf. 1983. The genetics of Type 1 (insulin-dependent) diabetes. Curr. Prob. Clin. Biochem. 12:45-55.

9. Tait, B. D., A. Boyle, S. Solty, T. Cunningham, T. Mandel, F. I. R. Martin, and T. Doran. 1984. DR4 related antisera pattern differences in insulin-dependent diabetes and rheumatoid arthritis. Tissue Antigens. 24:228-233.

10. Raum, D., Z. Awdeh, E. J. Yunis, C. A. Alper, and K. H. Gabbay. 1984. Extended major histocompatibility complex haplotypes in Type 1 diabetes mellitus. J. Clin. Invest. 74:449-454. 
11. Bach, F. H., S. Rich, J. Barbosa, and M. Segall. 1985. Insulindependent diabetes associated HLA-D region encoded determinants. Hum. Immunol. 12:59-64.

12. Cohen-Haguenauer, O., E. Robbins, C. Massart, M. Busson, I. Deschamps, J. Hors, J. M. Lalouel, J. Dausset, and D. Cohen. 1985. A systematic study of HLA class II- $\beta$ DNA restriction fragments in insulin-dependent diabetes mellitus. Proc. Natl. Acad. Sci. USA. 82:33353339.

13. Böhme, J., B. Carlsson, J. Wallin, E. Möller, B. Persson, P. A. Peterson, and L. Rask. 1986. Only one DQ $\beta$ restriction fragment pattern of each DR specificity is associated with insulin-dependent diabetes. J. Immunol. 137:941-947.

14. Festenstein, H., J. Awad, G. A. Hitman, S. Cutbush, A. V. Groves, P. Cassell, W. Ollier, and J. A. Sachs. 1986. New HLA DNA polymorphisms associated with autoimmune diseases. Nature (Lond.). 322:64-67.

15. Michelsen, B., and $\AA$. Lernmark. 1987. Molecular cloning of a polymorphic DNA endonuclease fragment associates insulin-dependent diabetes mellitus with HLA-DQ. J. Clin. Invest. 79:1144-1152.

16. Korman, A. J., J. M. Boss, T. Spies, R. Sorrentino, K. Okada, and J. L. Strominger. 1985. Genetic complexity and expression of human class II histocompatibility antigens. Immunol. Rev. 85:45-86.

17. Reinsmoen, N. L., and F. H. Bach. 1982. Five HLA-D clusters associated with HLA-DR4. Hum. Immunol. 4:249-258.

18. Christy, M., A. Green, B. Cristau, H. Kromann, J. Nerup, P. Platz, M. Thomsen, L. P. Ryder, and A. Svejgaard. 1979. Studies of the HLA system and insulin-dependent diabetes. Diabetes Care. 2:209214.

19. Platz, P., B. K. Jakobsen, N. Morling, L. P. Ryder, A. Svejgaard, M. Thomsen, M. Christy, H. Kromann, J. Benn, J. Nerup, and A. Green. 1981. HLA-D and -DR antigens in genetic analysis of insulin-dependent diabetes mellitus. Diabetologia. 21:108-115.

20. Rowe, J. R., E. M. Mickelson, J. A. Hansen, M. J. MacDonald, C. I. Allen, K. H. Gabbay, E. J. Yunis, and M. J. Sheehy. 1988. T cell-defined DR4 subtypes as markers for Type 1 diabetes. Hum. Immunol. 51-60.

21. Sheehy, M. J., F. B. Quintieri, S. Y. Yang, S. M. Alosco, Y. Matsui, E. J. Yunis, and K. H. Gabbay. 1984. HLA antigens of insulin-dependent diabetics. I. PLT colonies detecting Dw10 and a new class II determinant distinct from HLA-D, DR, MB(DC), MT, and SB. Tissue Antigens. 23:290-300.

22. Shaw, S., R. J. Duquesnoy, and P. L. Smith. 1981. Population studies of the HLA-linked SB antigens. Immunogenetics. 14:153-162.

23. Hansen, J. A., P. Martin, M. Kamoun, B. Nisperos, and E. D. Thomas. 1981. A supertypic HLA-DR specificity (DR4 + 5) defined by a murine monoclonal antibody. Hum. Immunol. 2:103-111.

24. Maeda, H. 1984. Mouse monoclonal antibody detects a new polymorphic Ia determinant other than HLA-DR antigen: a possible allele of DC-1. Tissue Antigens. 23:163-170.

25. Koning, F., J. Raghoebar, G. M. T. Schreuder, R. Schuurman, and H. Bruning. 1985. A monoclonal antibody detecting an HLADQw1 related determinant. Tissue Antigens. 26:100-109.

26. Schreuder, G. M. T., H. Maeda, F. Koning, and J. D'Amaro. 1986. TA10 and 2B3, two new alleles in the HLA-DQ region recognized by monoclonal antibodies. Hum. Immunol. 16:127-136.
27. Scharf, S. J., A. Friedmann, C. Brautbar, F. Szafer, L. Steinman, G. Horn, U. Gyllensten, and H. A. Erlich. 1988. HLA class II allelic variation and susceptibility to pemphigus vulgaris. Proc. Natl. Acad. Sci. USA. 85:3504-3508.

28. Scharf, S. J., C. M. Long, and H. A. Erlich. 1988. Sequence analysis of the HLA-DR $\beta$ and HLA-DQ $\beta$ loci from three Pemphigus vulgaris patients. Hum. Immunol. 22:61-69.

29. Woolf, B. 1955. On estimating the relationship between blood group and disease. Ann. Hum. Genet. 19:251-253.

30. Miettinen, O. 1976. Estimability and estimation in case-referent studies. Am. J. Epidemiol. 103:226-235.

31. Fisher, R. A. 1960. The Design of Experiments. Oliver \& Boyd, Edinburgh. 248 pp.

32. Fienberg, S. E. 1980. The Analysis of Cross-Classified Categorical Data. MIT Press, Cambridge, MA. 198 pp.

33. Maclaren, N. K., J. I. Rotter, W. J. Riley, C. M. Vadheim, W. Winter, D. Beckwith, and V. Henson. 1984. HLA-DR phenotypes and the absolute risks of insulin dependent diabetes. Presented at American Society for Histocompatibility and Immunogenetics.

34. Cavender, D. E., D. K. Wagener, B. S. Rabin, D. J. Becker, T. J. Orchard, M. S. Eberhardt, R. E. LaPorte, A. L. Drash, and L. H. Kuller. 1984. The Pittsburgh insulin-dependent diabetes mellitus (IDDM) study. HLA antigens and haplotypes as risk factors for the development of IDDM in IDDM patients and their siblings. J. Chron. Dis. 37:555-568.

35. Klein, R., B. E. K. Klein, S. E. Moss, D. L. DeMets, I. Kaufman, and P. S. Voss. 1984. Prevalence of diabetes mellitus in southern Wisconsin. Am. J. Epidemiol. 119:54-61.

36. Todd, J. A., J. I. Bell, and H. O. McDevitt. 1987. HLA-DQ $\beta$ gene contributes to susceptibility and resistance to insulin-dependent diabetes mellitus. Nature (Lond.). 329:599-604.

37. Horn, G., D. Bugawan, C. Long, and H. A. Erlich. 1988. Allelic sequence variation of the HLA-DQ loci: relationships to serology and to insulin-dependent diabetes susceptibility. Proc. Natl. Acad. Sci. USA. 85:6012-6016.

38. Nepom, G. T., J. Palmer, and B. S. Nepom. 1986. Specific HLA class II variants associated with IDDM. In The Immunology of Diabetes Mellitus. M. A. Jaworski, G. D. Molnar, R: V. Rajotte, and B. Singh, editors. Elsevier Science Publishers, Amsterdam. 9-20.

39. Gregersen, P. K., M. Shen, Q.-L. Song, P. Merryman, S. Degar, T. Seki, J. Maccari, D. Goldberg, H. Murphy, J. Schwenzer, C. Y. Wang, R. J. Winchester, G. T. Nepom, and J. Silver. 1986. Molecular diversity of HLA-DR4 haplotypes. Proc. Natl. Acad. Sci. USA. 83:2642-2646.

40. Nishimoto, H., H. Kikutani, K. Yamamura, and T. Kishimoto. 1986. Prevention of autoimmune insulitis by expression of I-E molecules in NOD mice. Nature (Lond.) 328:432-434.

41. Ambinder, J. M., N. Chiorazzi, A. Gibofsky, M. Fotino, and H. G. Kunkel. 1982. Special characteristics of cellular immune function in normal individuals of the HLA-DR3 type. Clin. Immunol. Immunopathol. 23:269-274.

42. MacDonald, M. J., J. Gottschall, J. B. Hunter, and K. L. Winter. 1986. HLA-DR4 in insulin-dependent diabetic parents and their diabetic offspring: A clue to dominant inheritance. Proc. Natl. Acad. Sci. USA. 83:7049-7053. 